\title{
Entry, Exit, and Imperfect Competition in the Long Run
}

\author{
Rabah Amir* and Val E. Lambson ${ }^{\dagger}$
}

Final version

Suggested running title: Imperfect Competition

${ }^{*}$ CORE and Economics Dept., Université Catholique de Louvain, 1348 Louvain-La-Neuve, Belgium; email: amir@core.ucl.ac.be

$\dagger$ (Corresponding author), Brigham Young University, Economics Dept., Provo UT 84602, USA; fax number: (801) 378-2844; email: vlambson@byu.edu 
Abstract: An infinite-horizon, stochastic model of entry and exit with sunk costs and imperfect competition is constructed. A subgame perfect Nash equilibrium for the general dynamic stochastic game is shown to exist as a limit of finite-horizon equilibria. This equilibrium has a relatively simple structure characterized by two numbers per finite history. Under very general conditions, it tends to exhibit excessive entry and insufficient exit relative to a social optimum. Journal of Economic Literature Classification Numbers: C73, D43, L13

Key Words and Phrases: Entry and Exit, Dynamic Games, Integer Constraints. 


\section{Introduction}

Accepting the proposition that firms are forward-looking, economists have constructed myriad dynamic models of industry evolution, that is, models that incorporate time and (sometimes) uncertainty. For tractability, many have been two-period models. While these have yielded important insights, they suffer from the well-known drawback that the final period behaves like a static (one-period) model. This is a potential problem because firms expecting static behavior in the near future may behave differently than firms expecting continued dynamic behavior. Circumventing this problem are the infinite-horizon models of industry evolution. However, most of these - e.g. Jovanovic [8], Dixit [4], Lambson [10], Rob [13], Hopenhayn [7], and Klepper [9]—posit infinitesimally-sized price-taking firms. Thus they illuminate competitive processes but are ill-suited for exploring traditional industrial organization questions regarding market structure and monopoly power. ${ }^{1}$

This paper formulates a stochastic infinite-horizon model where the number of active firms must be an integer. Entry and exit are endogenously generated by exogenous shocks — such as changes in demand or factor prices - that are external to the firms. The infinite horizon avoids the "final period problem" while the integer constraint makes the model suitable for addressing traditional industrial organization questions concerning imperfect competition.

Section 2 describes the model: a dynamic stochastic game in discrete time with countably many time periods and countably many firms. In each period, inactive firms choose whether to become active by paying an entry cost and active firms choose whether to become inactive by exiting and receiving a scrap value. The framework is very general. The one-period profits of active firms are modeled in reduced form, the only requirement being that perfirm profit be decreasing in the number of active firms. Furthermore, the stochastic process governing the exogenous shocks can be of any form — for example, it need not be Markovianas long some mild boundedness conditions are satisfied.

\footnotetext{
${ }^{1}$ An important exception is by Ericson and Pakes [5]. Their model is more general than ours in that it allows firm-level investment, but less general in that the restrictions placed on the primitives rule out deterministic processes and other cases of interest. (See Amir [1].) Another difference is that their existence proof employs an abstract fixed-point argument whereas ours is constructive.
} 
Section 3 proves that the game has a subgame perfect Nash equilibrium that is the limit of finite-horizon equilibria. The proof is constructive and provides a useful characterization of the equilibrium path.

Section 4 contains an example of how the framework may be applied: an analysis of the welfare implications of unregulated entry and exit. That there is a tendency for excessive entry in a two-stage model-where firms make entry decisions in the first stage and production decisions in the second stage - was established by Mankiw and Whinston [12]. The stochastic infinite-horizon model analyzed here allows a deeper investigation. For the class of equilibria constructed in Section 3, not only is there a tendency for excessive entry when times are good but there is also a tendency for insufficient exit when times are bad. Furthermore, these tendencies are not reversed in the present even when firms take into account that there will tend to be too many firms in the future. Thus, these equilibria tend to exhibit too many active firms in every period of every realization of the stochastic process. Section 4 closes with a reminder that there can be equilibria that are not limits of finite-horizon equilibria and that have very different properties. This reminder takes the form of an equilibrium in which entry falls more than one short of the optimal number of firms. Thus the tendency for an excessive number of firms in imperfectly competitive markets is not universal.

\section{The Model}

Suppose countably many firms, indexed by $i \in\{1,2,3, \ldots\}:=I$, exist for countably many periods indexed by $t \in\{1,2,3, \ldots\}$. Let $m_{t}$ be the market condition in period $t$. Specifically, $m_{t}$ lists all the relevant exogenous variables - e.g. factor prices, demand parameters, entry costs, scrap values, etc. - at time $t$. Let $h=\left(m_{1}, \ldots, m_{t}\right)$ be the market conditions in the first $t$ periods (to be called a $t$-period market history), let $H_{t}$ be the set of possible $t$-period market histories, and let $H=\cup_{t=1}^{\infty} H_{t}$ be the set of all possible finite market histories. Assume the set of possible market conditions is finite or countable, implying that $H$ is countable. Market conditions are governed by an exogenous stochastic process. Let $\rho(g \mid h)$ be the probability that the market history $g \in H_{\tau}$ is realized given that the market history $h \in H_{t}$ is realized, 
where $\tau \geq t$. Then $\rho$ induces a partial ordering $\geq$ on market histories: $g \geq h$ iff $\rho(g \mid h)>0$. If $g \geq h$ and $g \neq h$ then one can write $g>h$. If $h \leq f \leq g$ then one can write $f \in[h, g]$. If $h \in H_{t}$ and $t \geq 2$, then $h^{-1} \in H_{t-1}$ lists the first $t-1$ market conditions of $h$.

Finitely many (perhaps zero) firms are initially active and the others are initially inactive. At the beginning of each period, $t$, and given the market history $h \in H_{t}$, an inactive firm can enter (become active) by paying the entry cost $\xi_{h}$. Similarly, an active firm may exit (become inactive) and recoup the scrap value $\chi_{h}$ (which may be negative). Then active firms play a symmetric game (e.g. Cournot) yielding a current payoff of $\pi_{h}\left(y_{h}\right)$ to each active firm, where $y_{h}$ is the number of active firms. Note that $\xi_{h}, \chi_{h}$, and $\pi_{h}$ depend only on the current market condition rather than the entire market history, but it is convenient to use market history subscripts rather than market condition subscripts. Thus, for example, $\xi_{h}$ is understood to be the entry cost in the market condition prevailing in the last period of the market history $h$ (or the "entry cost at $h$ ").

Call $a_{0}^{i} \in\{0,1\}$ firm $i$ 's initial activity index and call $a_{h}^{i} \in\{0,1\}$ firm $i$ 's activity index at $h$; an index of 1 denotes activity and an index of 0 denotes inactivity. Call $a_{0}=\left\{a_{0}^{i}\right\}_{i \in I}$ and $a_{h}=\left\{a_{h}^{i}\right\}_{i \in I}$ the activity vectors initially and at $h$, respectively. A state is a pair $(m, a)$, interpreted as the current market condition and the previous period's activity vector. A $t$-period history, $s$, is a list of $t$ states. Let $S_{t}$ be the set of possible $t$-period histories and let $S=\cup_{t=1}^{\infty} S_{t}$ be the set of all possible finite histories. A strategy for firm $i$ is a decision rule $\sigma^{i}: S \rightarrow\{0,1\}$ that specifies firm $i$ 's activity index as a function of the history. Given a history $s \in S_{t}$, an induced strategy $\sigma_{s}^{i}$ is the restriction of $\sigma^{i}$ to histories that follow $s$ (in the sense that their first $t$ states are the list $s$ ). Let $\Sigma_{s}^{i}$ be the set of firm $i$ 's induced strategies given $s$. The induced strategy profile $\sigma_{s}=\left\{\sigma_{s}^{i}\right\}_{i \in I}$ induces a path, that is, a stochastic sequence of activity vectors $\left\{a_{g}\right\}_{g \geq h}$. Associated with that path is a value for firm $i$ given $s$ :

$$
\bar{V}_{s}^{i}\left(\sigma_{s}\right)=\sum_{\tau=t}^{\infty} \delta^{\tau-t} \sum_{g \geq h} \rho(g \mid h)\left\{-\max \left(0, a_{g}^{i}-a_{g^{-1}}^{i}\right) \xi_{g}+\max \left(0, a_{g^{-1}}^{i}-a_{g}^{i}\right) \chi_{g}+a_{g}^{i} \pi_{g}\left(y_{g}\right)\right\},
$$

where $\delta \in[0,1)$ is a discount factor. Equilibrium can now be formally defined.

Definition: A (subgame perfect Nash) equilibrium is a strategy profile $\widetilde{\sigma}=\{\widetilde{\sigma}\}_{i \in I}$ such that $\bar{V}_{s}^{i}\left(\widetilde{\sigma}_{s}\right) \geq \bar{V}_{s}^{i}\left(\sigma_{s}^{i}, \widetilde{\sigma}_{s}^{-i}\right)$ for all $i \in I$, for all $s \in S$, and for all $\sigma_{s}^{i} \in \Sigma_{s}^{i}$, where $\widetilde{\sigma}_{s}$ is the strategy profile induced by $\widetilde{\sigma}$ for each $s$ and where $\widetilde{\sigma}_{s}^{-i}$ is $\widetilde{\sigma}_{s}$ with the $i^{t h}$ element removed. 


\section{Existence of Equilibrium}

This section contains a heuristic sketch of a constructive proof that a subgame perfect Nash equilibrium exists. The formalities are relegated to the appendix. The constructed equilibrium is called a limit-lifo equilibrium because it is the limit of finite-horizon equilibria and the last firms to enter are the first to exit. A limit-lifo equilibrium is characterized by a collection of integer pairs, $\left\{N_{h}, X_{h}\right\}_{h \in H}$, one for each market history. Suppose $h \in H_{t}$ is the market history in period $t$. If there were fewer than $N_{h}$ active firms in period $t-1$ - that is, if $y_{h^{-1}}<N_{h}$-then there is entry and $y_{h}=N_{h}$. If $y_{h^{-1}}>X_{h}$ then there is exit and $y_{h}=X_{h}$. Otherwise, there is neither entry nor exit in period $t$ and $y_{h}=y_{h^{-1}}$. Thus the evolution of the number of firms is governed by

$$
y_{h}=\min \left\{X_{h}, \max \left\{N_{h}, y_{h^{-1}}\right\}\right\}
$$

To construct $\left\{N_{h}, X_{h}\right\}_{h \in H}$, integer pairs $\left\{N_{h}^{T}, X_{h}^{T}\right\}_{h \in \cup_{t=1}^{T} H_{t}}$ are constructed for each $T$-period truncation of the model by backward induction. Then a limiting argument is invoked as $T$ increases without bound. For each $T, t \leq T$, and each $h \in H_{t}, N_{h}^{T}$ (respectively, $X_{h}^{T}$ ) is the largest integer such that the expected present value (through period $T$ ) of the firm that will be the first to exit is not less than the entry cost (respectively, scrap value), given that the evolution of the number of firms is governed by

$$
y_{h}=\min \left\{X_{h}^{T}, \max \left\{N_{h}^{T}, y_{h^{-1}}\right\}\right\},
$$

which is the finite-horizon analogue to (3.1).

Unfortunately, $N_{h}^{T}$ and $X_{h}^{T}$ are not necessarily monotonic in $T$, making it impossible to verify that limits exist. Instead, a diagonalization argument is invoked so that $N_{h}$ and $X_{h}$ can be derived for each $h \in H$ as limits of an appropriately specified subsequence. Firms' strategies are then constructed so that the number of firms is governed by (3.1) and such that no firm ever has an incentive to deviate from its assigned strategy. This is done by partitioning the set of initially inactive firms into countably many countable subsets. Firms in each subset are ordered and each subset is assigned a market history. Each inactive firm considers entry only in the last period of its assigned market history; if its index is sufficiently 
low it enters. Active firms consider exit according to a last-in-first-out rule. Any firm that deviates from its assigned behavior by becoming or remaining active becomes the "marginal firm" - that is, the firm expected to exit first. Any firm that deviates from its assigned behavior by becoming or remaining inactive loses its chance at further equilibrium activity in the induced subgame.

Of course, some assumptions are required. First, the natural assumption that operating profits do not increase with the number of firms is imposed:

A1. For all $h \in H, \pi_{h}\left(y_{h}\right)$ is non-increasing in $y_{h}$.

Next, it is proper to allow $X_{h}$ to be infinite; this models situations where there is no exit regardless of the number of active firms (e.g. due to the absence of scrap values and fixed operating costs). However, it is desirable to bound entry in each period so that the number of active firms is always finite. This is accomplished as follows. For each $h \in H$, let $B_{h}$ be the collection of subsets of $H$ defined as follows. If $\beta_{h} \in B_{h}$, then (1) $g>h$ for all $g \in \beta_{h}$, and (2) if $g \in \beta_{h}$ and $g^{\prime} \in \beta_{h}$ then it is not the case that $g>g^{\prime}$. In words, all market histories in $\beta_{h}$ follow $h$ but not each other. Given $\beta_{h} \in B_{h}$, define

$$
\alpha_{h}=\left\{f \geq h \mid f<g \text { for some } g \in \beta_{h}\right\}
$$

In words, all market histories in $\alpha_{h}$ (weakly) follow $h$ but do not follow any market history in $\beta_{h}$. The next assumption can now be stated.

A2. For each $h \in H$ there exists a positive integer $\bar{y}_{h}$ such that, for all $y>\bar{y}_{h}$,

$$
\sup _{\beta_{h} \in B_{h}} \sum_{\tau=t}^{\infty} \delta^{\tau-t}\left[\sum_{g \in H_{\tau} \cap \alpha_{h}} \rho(g \mid h) \pi_{g}(y)+\sum_{g \in H_{\tau} \cap \beta_{h}} \rho(g \mid h) \chi_{g}\right]<\xi_{h} .
$$

Assumption A2 bounds the number of active firms at each market history $h$. Intuitively, if there are $y_{h}>\bar{y}_{h}$ active firms in the last period of market condition $h$ then the marginal firm - that is, the first of the $y_{h}$ firms to exit - cannot have an expected present value exceeding the left hand side of the inequality in A2. Since this is less than the entry cost, no more than $\bar{y}_{h}$ can enter in the last period of $h$. Thus, since $\bar{y}_{h}$ is finite for all $h \in H$, the number of active firms is always finite. It may, however, increase over time without bound. 
Finally, a technical assumption is required to ensure that expected present values converge as the horizon lengthens. This is accomplished by assumption A3:

A3. There exists $\kappa \in(\delta, 1]$ and $M>0$ such that, for all $t$ and for all $h \in H_{t}$, $\left|\pi_{h}(1)\right|<M / \kappa^{t}$ and $\left|\chi_{h}\right|<M / \kappa^{t}$.

The theorem can now be stated and rigorously proved. Note that the proof is constructive, and thus provides a characterization of an equilibrium. In particular, the evolution of the number of active firms over time is governed by (3.1).

Theorem 1: If A1-A3 are satisfied then a (subgame perfect Nash) equilibrium exists.

Proof: See appendix.

\section{Excessive Entry and Insufficient Exit}

The traditional industrial organization literature often assumed that there is insufficient entry in imperfectly competitive markets. However, Mankiw and Whinston [12] established a tendency for excessive entry in a simple two-stage framework where in the first stage firms make entry decisions and in the second stage active firms make production decisions. ${ }^{2}$ Our model allows a much stronger result. Limit-lifo equilibria not only exhibit a tendency for excessive entry when times are good but also a tendency for insufficient exit when times are bad. Furthermore, these tendencies are not reversed in the present even when firms take into account that there will tend to be too many firms in the future. Thus, equilibrium tends to exhibit too many active firms in every period of every realization of the stochastic process. $^{3}$ This theorem requires additional notation and assumptions - analogous to those used by Mankiw and Whinston-which will now be introduced.

Assume each market condition is completely described by a differentiable downwardsloping inverse demand function and a twice differentiable, convex cost function. Given

\footnotetext{
2 "Tendency" means that the number of active firms is never more than one firm short of, but can greatly exceed, the optimal number. That the number of firms can be one less than optimal is due to the integer constraint.

${ }^{3}$ This result contrasts with Rob's [13] continuum model where, due to learning externalities, the model exhibits insufficient entry.
} 
an initial number of active firms, let $\mathbf{y}^{*}=\left\{y_{h}^{*}\right\}_{h \in H}$ be the optimal stochastic sequence of numbers of firms when the social planner controls entry and exit but not the production and pricing decisions of active firms. Formally, $\mathbf{y}^{*}$ maximizes

$$
\begin{aligned}
W(\mathbf{y}) & :=\sum_{\tau=1}^{\infty} \sum_{h \in H_{\tau}} \rho_{h}\left\{\left[\int_{0}^{Q_{y_{h}}} P_{h}(s) d s-y_{h} c_{h}\left(q_{y_{h}}\right)\right]\right. \\
& \left.-\max \left\{0, y_{h}-y_{h^{-1}}\right\} \xi_{h}+\max \left\{0, y_{h^{-1}}-y_{h}\right\} \chi_{h}\right\} \\
& :=\sum_{\tau=1}^{\infty} \sum_{h \in H_{\tau}} \rho_{h}\left[S_{h}\left(y_{h}\right)-\max \left\{0, y_{h}-y_{h^{-1}}\right\} \xi_{h}+\max \left\{0, y_{h^{-1}}-y_{h}\right\} \chi_{h}\right]
\end{aligned}
$$

where $Q_{y_{h}}=y_{h} q_{h}$ is total output when there are $y_{h}$ firms at $h, P_{h}$ is the inverse demand function at $h, c_{h}$ is the individual firm's cost function at $h, q_{y_{h}}$ is the equilibrium per-firm output given $y_{h}$ firms at the history $h$ and $\rho_{h}$ is the unconditional probability of the market history $h$ occurring. $S_{h}\left(y_{h}\right)$ will be called the current surplus at $h$. For all $h$, assume (following Mankiw and Whinston):

A4. $Q_{y_{h}}>Q_{y_{h}^{\prime}}$ for all $y_{h}>y_{h}^{\prime}$ and $\lim _{y_{h} \rightarrow \infty} Q_{y_{h}}<\infty$.

A5. $q_{y_{h}}<q_{y_{h}^{\prime}}$ for all $y_{h}>y_{h}^{\prime}$.

A6. $P_{h}\left(Q_{y_{h}}\right)-c_{h}^{\prime}\left(q_{y_{h}}\right) \geq 0$ for all $y_{h}$.

In words, in each period of each realization, industry output increases and is bounded in the number of firms, per-firm output is decreasing in the number of firms, and price is no less than the marginal cost given the number of firms. These assumptions are not very restrictive: Amir and Lambson [2] provide (minimal) conditions sufficient for A4 and A5 to hold for Cournot equilibria. Specifically, defining $\Delta:=-P^{\prime}(Q)+c^{\prime \prime}(q)$, they show that the first part of A4-namely $Q_{y_{h}}>Q_{y_{h}^{\prime}}$ for all $y_{h}>y_{h}^{\prime}$-holds if $\Delta>0$ globally. ${ }^{4}$ This is a very general condition, implied in particular by our assumption that $P$ is downward-sloping and $c$ is convex. They also show that A5 holds if $\Delta>0$ globally and $\log P$ is a concave function, which still covers most examples of interest. On the other hand, they show that if $\Delta<0$ globally (which requires a strongly concave cost function $c$ ), the opposite of the first part of A4 holds. Also, with $\Delta>0$, A5 may fail if $\log P$ is convex. Thus, while A4 and A5 are not universal, they are satisfied under very broad conditions covering most cases of interest in Cournot models. A6 follows from the best reply property of Cournot equilibria.

\footnotetext{
${ }^{4}$ The second part of A4-namely, $\lim _{y_{h} \rightarrow \infty} Q_{y_{h}}<\infty$-holds, for example, if $P(Q)<c^{\prime}(0)$ for some $Q$.
} 
Given an initial number of active firms, let $\mathbf{y}^{e}=\left\{y_{h}^{e}\right\}_{h \in H}$ be a stochastic limit-lifo equilibrium sequence of numbers of firms. The theorem asserts that the equilibrium number of firms never falls short of the optimal number by more than one. It is not difficult to construct examples where the equilibrium number of firms greatly exceeds the optimal number.

Theorem 2: For all $h \in H, y_{h}^{e} \geq y_{h}^{*}-1$.

Proof: See appendix.

By construction, a limit-lifo equilibrium is the limit of finite-horizon equilibria. It is well known that infinite-horizon games typically exhibit equilibria that are not limit equilibria, that is, not limits of finite-horizon equilibria.. (See, for example, Fudenberg and Tirole [6].) In spite of this, most studies of dynamic games restrict attention to limit equilibria. This point is discussed in detail by Basar and Olsder [3] for the widely-employed class of linear-quadratic games. Also, Tsutsui and Mino [14] provide a specific example of an infinite-horizon equilibrium that is not a limit of finite-horizon equilibria in a continuous-time (differential) game.

The following - due to an anonymous contributor - is a reminder that restricting attention to limit equilibria is not without loss of generality in our framework either. In particular, the Mankiw-Whinston tendency for excessive entry need not hold. Suppose the same Cournot game is repeated in all periods, where inverse demand is $P=1-Q$ and production costs are zero. Thus per-firm operating profit in a period with $y$ active firms is $\pi(y)=\left(\frac{1}{y+1}\right)^{2}$. Suppose $\xi=2, \chi=1.7$, and $\delta=.99$. It is straightforward to establish that a social planner would maximize discounted producer and consumer surplus (subject to Cournot behavior) by having three firms enter in the first period and allowing no further entry or exit. There exist equilibria, however, that exhibit no entry at all along the equilibrium path. One of the simplest is defined as follows. Given $a_{t-1}$, let $\iota$ be the index of the active firm at time $t-1$ with the largest index; formally, $\iota=\max _{i \in\left\{i \mid a_{t-1}^{i}=1\right\}} i$. (If there are infinitely many active firms then $\iota=\infty$.) Let $\# a_{t}$ be the number of active firms in period $t$ and set $\# a_{0}=0$. For each $i$, define firm $i$ 's strategy as follows: for each $t, a_{t}^{i}=1$ if $\# a_{t-1}=6$ and $a_{t-1}^{i}=1$, or if $\# a_{t-1} \notin\{0,6, \infty\}$ and $i \in\{\iota+1, \ldots, \iota+6\}$, or if $\# a_{t-1}=\infty$ and $i \in\{1, \ldots, 6\} ; a_{t-1}^{i}=0$ otherwise. Intuitively, since $\pi(6) /(1-\delta)>\xi>\chi>\pi(7) /(1-\delta)$, there are subgame perfect Nash equilibria exhibiting entry by six firms and no further entry or exit. These equilibria 
can be used as "threats" to dissuade all entry. Specifically, given the strategies of the other firms, if a firm ever enters it will enjoy monopoly profit for one period but will be joined thereafter by six new firms; it will thus find it optimal to exit without having recouped its entry cost.

\section{Appendix}

Theorem 1: If A1-A3 are satisfied then a (subgame perfect Nash) equilibrium exists.

Proof: For each positive integer $T$ and each market history $h \in H$, define two integers, $N_{h}^{T}$ and $X_{h}^{T}$ as follows. For $t>T$ and $h \in H_{t}$, let $N_{h}^{T}=X_{h}^{T}=0$. For $h \in H_{T}$, define $N_{h}^{T}$ and $X_{h}^{T}$ as the largest integer values of $y_{h}$ satisfying $\pi_{h}\left(y_{h}\right)-\xi_{h} \geq 0$ and $\pi_{h}\left(y_{h}\right)-\chi_{h} \geq 0$, respectively, if well-defined. Otherwise, if $\pi_{h}(1)-\xi_{h}<0$ let $N_{h}^{T}=0$; if $\pi_{h}(1)-\chi_{h}<0$ let $X_{h}^{T}=0$; and if $\pi_{h}\left(y_{h}\right)-\chi_{h} \geq 0$ for all $y_{h}$ let $X_{h}^{T}=\infty$. (A2 implies $N_{h}^{T}$ is finite.) For $t<T$, and having defined $N_{g}^{T}$ and $X_{g}^{T}$ for all $g \in H_{\tau}$ and all $\tau>t$, define $N_{h}^{T}$ and $X_{h}^{T}$ for each $h \in H_{t}$ as the largest integer values of $y_{h}$ that satisfy, respectively,

$$
V_{h}^{T}\left(y_{h}\right):=\sum_{\tau=t}^{T} \delta^{\tau-t}\left[\sum_{g \in H_{\tau} \cap \Theta} \rho(g \mid h) \pi_{g}\left(y_{g}\right)+\sum_{g \in H_{\tau} \cap \Phi} \rho(g \mid h) \chi_{g}\right] \geq \xi_{h}
$$

and

$$
V_{h}^{T}\left(y_{h}\right):=\sum_{\tau=t}^{T} \delta^{\tau-t}\left[\sum_{g \in H_{\tau} \cap \Theta} \rho(g \mid h) \pi_{g}\left(y_{g}\right)+\sum_{g \in H_{\tau} \cap \Phi} \rho(g \mid h) \chi_{g}\right] \geq \chi_{h}
$$

where

$$
\begin{gathered}
\Theta:=\left\{g \geq h \mid y_{f} \geq y_{h} \forall f \in[h, g]\right\} \\
\Phi:=\left\{g>h \mid g^{-1} \in \Theta \text { and } y_{g}<y_{h}\right\}
\end{gathered}
$$

and

$$
y_{g}=\min \left\{X_{g}^{T}, \max \left\{N_{g}^{T}, y_{g^{-1}}\right\}\right\} .
$$

If $N_{h}^{T}$ or $X_{h}^{T}$ is not well-defined in this way then proceed as follows. If $V_{h}^{T}(1)-\xi_{h}<0$ let $N_{h}^{T}=0$; if $V_{h}^{T}(1)-\chi_{h}<0$ let $X_{h}^{T}=0$; and if $V_{h}^{T}\left(y_{h}\right)-\chi_{h} \geq 0$ for all $y_{h}$ let $X_{h}^{T}=\infty$. Note 
that $\Theta, \Phi$, and the $y_{g}$ values all depend on $y_{h}$, which dependency is suppressed for notational clarity. The set $\Theta$ contains those future market histories before any of the active firms at $h$ exit. The set $\Phi$ contains those future histories at which, for the first time, some firm that was active at $h$ exits. Thus $V_{h}^{T}\left(y_{h}\right)$ is interpretable as the value of the marginal firm, that is, the active firm at $h$ that will exit first.

Now index $H$ by the positive integers and let $g(j)$ be the market history assigned to the integer $j$. Consider the sequences $\left\{N_{g(1)}^{T}\right\}_{T \in I}$ and $\left\{X_{g(1)}^{T}\right\}_{T \in I}$. Since $\left\{N_{g(1)}^{T}\right\}_{T \in I}$ is bounded (by A2), there exists $I_{1}=\left\{T_{11}, T_{12}, \ldots\right\} \subset I$ such that the subsequences $\left\{N_{g(1)}^{T}\right\}_{T \in I_{1}}$ and $\left\{X_{g(1)}^{T}\right\}_{T \in I_{1}}$ converge to, say, $N_{g(1)}$ and $X_{g(1)}$, respectively (where $X_{g(1)}$ may be infinite). Having defined $I_{j-1}$, define $I_{j}=\left\{T_{j 1}, T_{j 2}, \ldots\right\} \subset I_{j-1}$ so the subsequences $\left\{N_{g(j)}^{T}\right\}_{T \in I_{j}}$ and $\left\{X_{g(j)}^{T}\right\}_{T \in I_{j}}$ converge to, say, $N_{g(j)}$ and $X_{g(j)}$, respectively (where $X_{g(j)}$ may be infinite). Finally, define the diagonal sequence $\bar{I}=\left\{T_{11}, T_{22}, \ldots\right\}$. Then, by construction, $\left\{N_{g}^{T}\right\}_{T \in \bar{I}}$ and $\left\{X_{g}^{T}\right\}_{T \in \bar{I}}$ converge to $N_{g}$ and $X_{g}$, respectively, for all $g \in H$ (where $X_{g}$ may be infinite).

Note that, by construction, $V_{h}^{T}\left(N_{h}^{T}\right) \geq \xi_{h}>V_{h}^{T}\left(N_{h}^{T}+1\right)$ and $V_{h}^{T}\left(X_{h}^{T}\right) \geq \chi_{h}>V_{h}^{T}\left(X_{h}^{T}+1\right)$ for all $h \in H$ and for all $T$ if $N_{h}^{T}$ and $X_{h}^{T}$ are positive and finite. Thus, by A3, if $N_{h}$ and $X_{h}$ are both positive and finite then $V_{h}\left(N_{h}\right) \geq \xi_{h} \geq V_{h}\left(N_{h}+1\right)$ and $V_{h}\left(X_{h}\right) \geq \chi_{h} \geq V_{h}\left(X_{h}+1\right)$ for all $h \in H$, where for all $h \in H$,

$$
V_{h}\left(y_{h}\right):=\sum_{\tau=t}^{\infty} \delta^{\tau-t}\left[\sum_{g \in H_{\tau} \cap \Theta} \rho(g \mid h) \pi_{g}\left(y_{g}\right)+\sum_{g \in H_{\tau \cap \Phi}} \rho(g \mid h) \chi_{g}\right]
$$

where $\Theta$ and $\Phi$ are as before and $y_{g}=\min \left\{X_{g}, \max \left\{N_{g}, y_{g^{-1}}\right\}\right\}$. If $X_{h}$ is infinite then $V_{h}\left(y_{h}\right) \geq \chi_{h}$ for all $y_{h}$.

Construct firms' strategies as follows. Let $\left\{I_{h}\right\}_{h \in H}$ partition the set of initially inactive firms into countably infinitely many countably infinite subsets. Intuitively, $I_{h}$ is the set of firms assigned to consider entry at the market history $h$. Order the $y_{0}$ initially active firms by their indexes $i$ and assign them the integers from 1 to $y_{0}$. Let $\iota_{0}(i)$ be the integer initially assigned to the initially active firm $i$. Now, given a path of activity vectors, $\left\{a_{g}\right\}_{g \leq h}$, and having defined $\iota_{h^{-1}}(i)$ for each active firm at $h^{-1}$, define $\iota_{h}(i)$ for each active firm at $h$ as follows. First order the active firms at $h$ that were also active at $h^{-1}$ by $\iota_{h^{-1}}(i)$, then order new entrants that belong to the set $I_{h}$ by their indexes $i$, and finally order new entrants that 
do not belong to the set $I_{h}$ by their index numbers $i$. (A new entrant is a firm for which $a_{h}^{i}-a_{h^{-1}}^{i}=1$.) Having ordered the active firms at $h$, assign the positive integers between 1 and $y_{h}$ to them and let $\iota_{h}(i)$ be the integer assigned to the active firm $i$ at $h$. For each initially inactive firm, define

$$
\eta^{i}:=\sum_{j \in I_{h(i)} \cap\{j \mid j<i\}}\left(1-a_{h(i)^{-1}}^{j}\right)
$$

where $h(i)$ is the history such that $i \in I_{h(i)}$. Thus $\eta^{i}$ is the number of firms in $I_{h(i)}$ with lower indexes than firm $i$ that are not active coming into the last period of the market history $h(i)$. Firm $i$ 's equilibrium strategy $\widetilde{\sigma}^{i}$ is given by

$$
\begin{aligned}
& \text { If } a_{h^{-1}}^{i}=0 \text { then } \widetilde{a}_{h}^{i}=1 \text { iff } h=h(i) \text { and } N_{h}-y_{h^{-1}}>\eta^{i} . \\
& \text { If } a_{h^{-1}}^{i}=1 \text { then } \widetilde{a}_{h}^{i}=1 \text { iff } X_{h} \geq \iota_{h}(i) .
\end{aligned}
$$

Note that the construction of the strategies guarantees that

$$
y_{g}=\min \left\{X_{g}, \max \left\{N_{g}, y_{g^{-1}}\right\}\right\}
$$

for all $g \in H$ and that the most recent entrant is the first to exit. Thus the marginal firm's value - that is, the value of the active firm at $h$ that will be the first to exit - is given by $V_{h}\left(y_{h}\right)$ along the equilibrium path.

Now consider all possible one-shot deviations at an arbitrary history $s$. First suppose an inactive firm deviates by entering when its equilibrium strategy dictates otherwise. By construction, it will be designated as the marginal firm and will earn $V_{h}\left(\widetilde{y}_{h}+1\right)-\xi_{h} \leq 0$ where $\widetilde{y}_{h}$ is the equilibrium number of firms at $h$. Next suppose an inactive firm deviates by not entering when it is supposed to. It then receives a payoff of zero, which is no greater than the $V_{h}\left(\widetilde{y}_{h}\right)-\xi_{h} \geq 0$ it would gain by following its equilibrium strategy. Next suppose an active firm exits when its equilibrium strategy dictates otherwise. It then receives a payoff of $\chi_{h}$ which cannot exceed its equilibrium value of $V_{h}\left(\widetilde{y}_{h}\right) \geq \chi_{h}$. Finally, suppose an active firm does not exit when its strategy dictates to exit. Then it will be designated as the marginal firm and will have a value of $V_{h}\left(\widetilde{y}_{h}+1\right) \leq \chi_{h}$, which cannot exceed its payoff of $\chi_{h}$ for exiting. It follows that no one-shot deviation from equilibrium - and hence no deviation 
from equilibrium - is profitable. Thus the constructed strategy profile constitutes a subgame perfect Nash equilibrium. Q.E.D.

Theorem 2: For all $h \in H, y_{h}^{e} \geq y_{h}^{*}-1$.

Proof: First note that, for all $h \in H$ and for all $y_{h}>1$,

$$
\begin{aligned}
S_{h}\left(y_{h}\right)-S_{h}\left(y_{h}-1\right) & =\int_{Q_{y_{h}-1}}^{Q_{y_{h}}} P_{h}(s) d s-y_{h} c_{h}\left(q_{y_{h}}\right)+\left(y_{h}-1\right) c_{h}\left(q_{y_{h}-1}\right) \\
& =P_{h}\left(Q_{y_{h}-1}\right) q_{y_{h}-1}-c_{h}\left(q_{y_{h}-1}\right) \\
& -\left[P_{h}\left(Q_{y_{h}-1}\right) q_{y_{h}-1}-\int_{Q_{y_{h}-1}}^{Q_{y_{h}}} P_{h}(s) d s+y_{h}\left[c_{h}\left(q_{y_{h}}\right)-c_{h}\left(q_{y_{h}-1}\right)\right]\right] \\
& :=\pi_{h}\left(y_{h}-1\right)-M_{h}^{y_{h}} .
\end{aligned}
$$

Assumptions A4-A6 imply $M_{h}^{y_{h}}>0$, because

$$
\begin{aligned}
M_{h}^{y_{h}} & :=P_{h}\left(Q_{y_{h}-1}\right) q_{y_{h}-1}-\int_{Q_{y_{h}-1}}^{Q_{y_{h}}} P_{h}(s) d s+y_{h}\left[c_{h}\left(q_{y_{h}}\right)-c_{h}\left(q_{y_{h}-1}\right)\right] \\
& \geq P_{h}\left(Q_{y_{h}-1}\right) q_{y_{h}-1}-P_{h}\left(Q_{y_{h}-1}\right)\left[Q_{y_{h}}-Q_{y_{h}-1}\right]+y_{h}\left[c_{h}\left(q_{y_{h}}\right)-c_{h}\left(q_{y_{h}-1}\right)\right] \\
& \geq P_{h}\left(Q_{y_{h}-1}\right) q_{y_{h}-1}-P_{h}\left(Q_{y_{h}-1}\right)\left[Q_{y_{h}}-Q_{y_{h}-1}\right]+y_{h} c_{h}^{\prime}\left(q_{y_{h}-1}\right)\left[q_{y_{h}}-q_{y_{h}-1}\right] \\
& =\left[P_{h}\left(Q_{y_{h}-1}\right)-c_{h}^{\prime}\left(q_{y_{h}-1}\right)\right] y_{h}\left[q_{y_{h}-1}-q_{y_{h}}\right] \\
& >0 .
\end{aligned}
$$

The first line is definitional, the second is from A4 and $P^{\prime}<0$, the third is from $c^{\prime \prime} \geq 0$, the fourth is from $Q_{y} / y=q_{y}$ (symmetry), and the fifth is from A5 and A6.

Consider the $T$-period truncation of the model. Let $\mathbf{y}^{*}(T)$ be the optimal stochastic sequence of numbers of firms for the $T$-period truncation of the model; that is, $\mathbf{y}^{*}(T)$ maximizes

$$
W_{T}(\mathbf{y}):=\sum_{\tau=1}^{T} \sum_{h \in H_{\tau}} \rho_{h}\left[S_{h}\left(y_{h}\right)-\max \left\{0, y_{h}-y_{h^{-1}}\right\} \xi_{h}+\max \left\{0, y_{h^{-1}}-y_{h}\right\} \chi_{h}\right] .
$$

The strategy of proof is as follows. First show, by backward induction, that $y_{h}^{e}(T) \geq y_{h}^{*}(T)-1$ for all $h \in H_{t}$, all $t \leq T$, and all $T$. Then limiting arguments as $T \rightarrow \infty$ establish $y_{h}^{e} \geq y_{h}^{*}-1$ for all $h \in H$. 
Consider an arbitrary $T$ and arbitrary $h \in H_{T}$. If $y_{h}^{*} \leq 1$, then it is trivial that $X_{h}^{T} \geq N_{h}^{T} \geq y_{h}^{*}-1$. If $y_{h}^{*} \geq 2$ then let $\mathbf{y}$ be the truncated path that is identical to $\mathbf{y}^{*}$ except that $y_{h}=y_{h}^{*}-1$. By the optimality of $\mathbf{y}^{*}$,

$$
\begin{aligned}
W_{T}\left(\mathbf{y}^{*}\right)-W_{T}(\mathbf{y}) & =\delta^{T-1}\left[S_{h}\left(y_{h}^{*}\right)-S_{h}\left(y_{h}^{*}-1\right)-\gamma_{h}\right] \\
& =\delta^{T-1}\left[\pi_{h}\left(y_{h}^{*}-1\right)-M_{h}^{y_{h}^{*}}-\gamma_{h}\right] \\
& \geq 0
\end{aligned}
$$

where $\gamma_{h}=\xi_{h}$ if $y_{h}^{*}>y_{h^{-1}}^{*}$ and $\gamma_{h}=\chi_{h}$ otherwise. Since $M_{h}^{y_{h}^{*}}>0$, this implies $\pi_{h}\left(y_{h}^{*}-1\right)>$ $\gamma_{h}$. Thus, $X_{h}^{T} \geq y_{h}^{*}-1$ and, furthermore, if $y_{h}^{*}>y_{h^{-1}}^{*}$ then $N_{h}^{T} \geq y_{h}^{*}-1$.

Now consider $h \in H_{t}$ for $t<T$ and make the induction hypothesis that, for all $g>h$ in the truncated model, $X_{g}^{T} \geq y_{g}^{*}-1$ and, furthermore, that if $y_{g}^{*}>y_{g^{-1}}^{*}$ then $N_{g}^{T} \geq y_{g}^{*}-1$. If $y_{h}^{*} \leq 1$, then it is trivial that $X_{h}^{T} \geq N_{h}^{T} \geq y_{h}^{*}-1$. If $y_{h}^{*} \geq 2$ then consider the path $\mathbf{y}$ that is identical to $\mathbf{y}^{*}$ except $y_{h}=y_{h}^{*}-1$ and $y_{g}=\min \left\{X_{g}^{T}\right.$, $\left.\max \left\{N_{g}^{T}, y_{g^{-1}}\right\}\right\}$ for $g>h$; in words, $\mathbf{y}$ follows the optimal path until $h$, exhibits one less firm at $h$, and then is induced by the equilibrium strategies thereafter. Define three sets:

$$
\begin{gathered}
A=\left\{g \geq h \mid y_{f}=y_{h}^{*}-1 \text { and } y_{f}^{*}=y_{h}^{*} \forall \mathrm{f} \in[h, g]\right\} \\
B=\left\{g \notin A \mid g^{-1} \in A \text { and } y_{g}^{*} \geq y_{h}^{*}\right\} \\
C=\left\{g \notin A \mid g^{-1} \in A \text { and } y_{g}^{*}<y_{h}^{*}\right\} .
\end{gathered}
$$

The first set, $A$, is the set of histories exhibiting no change in either path from the number of firms at $h$. The second and third sets together are the set of "first times" that either of the paths exhibits entry or exit; $B$ is the set of such "first times" that exhibits no exit along the optimal path (and hence exhibits entry or exit along the path $\mathbf{y}$ ) and $C$ is the set of such "first times" that exhibits exit along the optimal path.

If $g \in B$ then either $y_{g}^{*}>y_{h}^{*}=y_{g^{-1}}^{*}$ or $y_{g}^{*}=y_{h}^{*}=y_{g^{-1}}^{*}$. If $y_{g}^{*}>y_{g^{-1}}^{*}$ then, by the induction hypothesis, $N_{g}^{T} \geq y_{g}^{*}-1>y_{g^{-1}}$; thus $V_{g}^{T}\left(y_{g}\right) \geq \xi_{g}$ (because, intuitively, there is entry along the path $\mathbf{y}$ induced by equilibrium strategies). If $y_{g}^{*}=y_{g^{-1}}^{*}$ and $g \in B$ then 
$y_{g} \neq y_{g^{-1}}=y_{g}^{*}-1$. Since, by the induction hypothesis, $y_{g}^{*}-1 \leq X_{g}^{T}$, it must be that $y_{g}>y_{g^{-1}}$. Thus $y_{g}=N_{g}^{T}$ and $V_{g}^{T}\left(y_{g}\right) \geq \xi_{g}$. Thus $V_{g}^{T}\left(y_{g}\right) \geq \xi_{g}$ for all $g \in B$. Furthermore, it is trivial that $V_{g}^{T}\left(y_{g}\right) \geq \chi_{g}$ for all $g \in C$.

Now define the path $\mathbf{y}^{\prime}$ for the truncated model by $y_{g}^{\prime}=y_{h}^{*}-1$ for $g \in A$ and $y_{g}^{\prime}=y_{g}^{*}$ otherwise. By the optimality of $\mathbf{y}^{*}$,

$$
\begin{aligned}
& W_{T}\left(\mathbf{y}^{*}\right)-W_{T}\left(\mathbf{y}^{\prime}\right) \\
& =\sum_{\tau=t}^{T} \delta^{\tau-t}\left[\sum_{g \in A_{\tau}} \rho(g \mid h) \pi_{g}\left(y_{h}^{*}-1\right)-M_{g}^{y_{h}^{*}}+\sum_{g \in B_{\tau}} \rho(g \mid h) \xi_{g}+\sum_{g \in C_{\tau}} \rho(g \mid h) \chi_{g}\right]-\gamma_{h} \\
& \geq 0
\end{aligned}
$$

where $\gamma_{h}=\xi_{h}$ if $y_{h}^{*}>y_{h^{-1}}^{*}, \gamma_{h}=\chi_{h}$ otherwise, and where $A_{\tau}:=A \cap H_{\tau}, B_{\tau}:=B \cap H_{\tau}$, and $C_{\tau}:=C \cap H_{\tau}$. Since $M_{g}^{y_{h}^{*}}>0$ for all $g$, this implies

$$
\begin{aligned}
\sum_{\tau=t}^{T} \delta^{\tau-t} \sum_{g \in A_{\tau}} \rho(g \mid h) \pi_{g}\left(y_{h}^{*}-1\right) & \\
& \geq \gamma_{h}-\sum_{\tau=t}^{T} \delta^{\tau-t} \sum_{g \in B_{\tau}} \rho(g \mid h) \xi_{g}-\sum_{\tau=t}^{T} \delta^{\tau-t} \sum_{g \in C_{\tau}} \rho(g \mid h) \chi_{g}
\end{aligned}
$$

By definition

$$
\begin{aligned}
V_{h}^{T}\left(y_{h}^{*}-1\right) & =\sum_{\tau=t}^{T} \delta^{\tau-t}\left[\sum_{g \in A_{\tau}} \rho(g \mid h) \pi_{g}\left(y_{h}^{*}-1\right)+\sum_{g \in B_{\tau} \cup C_{\tau}} \rho(g \mid h) V_{g}^{T}\left(y_{g}\right)\right] \\
& \geq \sum_{\tau=t}^{T} \delta^{\tau-t}\left[\sum_{g \in A_{\tau}} \rho(g \mid h) \pi_{g}\left(y_{h}^{*}-1\right)+\sum_{g \in B_{\tau}} \rho(g \mid h) \xi_{g}+\sum_{g \in C_{\tau}} \rho(g \mid h) \chi_{g}\right]
\end{aligned}
$$

where $y_{g}=\min \left\{X_{g}^{T}, \max \left\{N_{g}^{T}, y_{g^{-1}}\right\}\right\}$. The previous two inequalities together imply

$$
V_{h}^{T}\left(y_{h}^{*}-1\right) \geq \gamma_{h}
$$

Thus, $X_{h}^{T} \geq y_{h}^{*}-1$ and, furthermore, if $y_{h}^{*}>y_{h^{-1}}^{*}$ then $N_{h}^{T} \geq y_{h}^{*}-1$. Thus, by induction, for all $h \in H_{t}$ and $t \leq T, X_{h}^{T} \geq y_{h}^{*}-1$ and, furthermore, if $y_{h}^{*}>y_{h^{-1}}^{*}$ then $N_{h}^{T} \geq y_{h}^{*}-1$. 
With this result in hand it follows immediately that, for the same initial conditions, the equilibrium path satisfying $y_{h}^{e}=\min \left\{X_{h}^{T}, \max \left\{N_{h}^{T}, y_{h^{-1}}^{e}\right\}\right\}$ will always satisfy $y_{h}^{e} \geq y_{h}^{*}-1$ in the truncated model. Intuitively, when there is entry along the optimal path there will be at least $N_{h}^{T} \geq y_{h}^{*}-1$ firms on the equilibrium path (because $y_{h}^{e} \geq N_{h}^{T}$ for all $h$ ). Thus $y_{h}^{e}<y_{h}^{*}-1$ could only arise if there is exit along the equilibrium path; but if there is exit at $h$ along the equilibrium path then $y_{h}^{e}=X_{h}^{T} \geq y_{h}^{*}-1$.

Thus, for all $T$, the result holds for the truncated model. Taking limits (using diagonalization arguments as for Theorem 1) then establishes the result. Q.E.D.

\section{References}

[1] R. Amir, Continuous Stochastic Games of Capital Accumulation with Convex Transitions, Games Econ. Behav. 15 (1996), 111-131.

[2] R. Amir and V. E. Lambson, On the Effects of Entry in Cournot Markets, Rev. Econ. Stud. 67 (2000), 235-254.

[3] T. Basar and G. Olsder, Dynamic Noncooperative Game Theory, SIAM Classics, 1999.

[4] A. Dixit, Entry and Exit Decisions Under Uncertainty, J. Polit. Economy 97 (1989), 620-638.

[5] R. Ericson and A. Pakes, Markov-Perfect Industry Dynamics: A Framework for Empirical Work, Rev. Econ. Stud. 62 (1995), 53-82.

[6] D. Fudenberg and J. Tirole, Game Theory, MIT Press, (1991).

[7] H. Hopenhayn, Entry, Exit, and Firm Dynamics in Long Run Equilibrium, Econometrica 60 (1992), 127-1150.

[8] B. Jovanovic, Selection and the Evolution of Industry, Econometrica 50 (1982), 649-670.

[9] S. Klepper, Entry, Exit, Growth, and Innovation over the Product Cycle, Amer. Econ. Rev. 86 (1996), 562-583. 
[10] V.E. Lambson, Industry Evolution with Sunk Costs and Uncertain Market Conditions, Int. J. Ind. Organ. 6 (1991), 263-271.

[11] V.E. Lambson, Competitive Profits in the Long Run, Rev. Econ. Stud. 59 (1992), 125142.

[12] G. Mankiw and M. Whinston, Free Entry and Social Inefficiency, RAND J. Econ. 17 (1986), 48-58.

[13] R. Rob, Learning and Capacity Expansion Under Demand Uncertainty, Rev. Econ. Stud. 58 (1991), 655-675.

[14] S. Tsutsui and K. Mino, Nonlinear Strategies in Dynamic Duopolistic Competition with Sticky Prices, J. Econ. Theory 52 (1990), 136-61. 